\title{
AC 2007-2389: REINVENTING HOME AUTOMATION: A RELIABLE, COST EFFECTIVE APPROACH
}

\section{Chad Lloyd, Middle Tennessee State University}

Mr. Lloyd earned his Masters Degree in Engineering Technology from Middle Tennessee State University in 2006. The above paper is partially based on his thesis research. Mr. Lloyd has also earned an M.S. degree in Computer Science and a B.S. degree in Mass Communications from Middle Tennessee State University. He is currently working as a networking engineer for a large law firm in Nashville, Tennessee. His interests are wide spread in the technical field of embedded systems and PC-based control applications.

\section{Saleh Sbenaty, Middle Tennessee State University}

Dr. Sbenaty is currently a Professor of Engineering Technology at Middle Tennessee State University. He received the BS degree in EE from Damascus University and the MS and Ph.D. degrees in EE from Tennessee Technological University. He is actively engaged in curriculum development for technology education. He has written and co-authored several industry-based case studies. He is also conducting research in the area of mass spectrometry, power electronics, lasers, and instrumentation. 


\title{
Reinventing Home Automation: A Reliable, Cost Effective Approach
}

\begin{abstract}
Home automation is an exciting area of technology that is gaining momentum every day. Current implementations of home automation systems often utilize devices that are designed for other uses. A common example of this includes the use of a lighting dimmer that has the capacity of a 1000 watt load while only driving one or two 100 watt light bulbs. Other examples include inferior wireless and power line communication products that result in unreliable automation systems.

The current paper focuses on an implementation that features a modular design, allowing the planner to select only the modules needed to complete the installation. Furthermore, the system is completely hardwired and software configurable. Hence, making changes to the automation system is quick and simple. The paper includes design considerations, firmware listings, and schematics related to its modules as well as a discussion related to the client software that is resident on the host PC. The entire system is open-source, allowing for the home automation development community to make improvements and changes at will.
\end{abstract}

\section{Introduction}

Many home automation systems rely on wireless techniques and others use devices that were intended for other purposes. Examples of this include the use of X10 series of lamp modules and switches that communicate by "piggybacking" over AC wiring. Another example involves the use of dimmers in residential dwellings. This is an oversize usage when 1000 watts or more per channel designed for theatrical environments is used in residential settings. Besides, home automation is much too critical to depend on wireless technology for lighting control. It is also not necessary to size a single circuit for 1000 watts when the lighting device is rated much less.

This paper offers an alternative technique that includes a centralized point at which all high voltage lighting circuit lines terminate. All remaining lines used for light switches, sensors, and other devices are low voltage and are routed through a computer system.

A few of the benefits of this approach include:

1. Configuration by Software: The connections of light switches to lighting circuits are configured by a point and click interface. This allows for quick and easy configuration.

2. Automatic, Pre-emptive Lighting: Sensors can detect the presence of someone in the room and automatically turn on the light to the appropriate level. Sensors can also light up the path when someone walks throughout the house.

3. Soft On, Soft Off: Depending on the program, by turning on the light switch at night, the light will illuminate slowly. During daytime, however, the light will immediately illuminate. 
For instance, early in the morning, a bathroom light can immediately light up to $15 \%$, then gradually up to $100 \%$ over the course of 1 minute, allowing one's eyes to adjust.

4. Integrated Burglar Alarm: The integrated burglar alarm is a full-featured system with zoning capabilities, follower and delay settings.

5. Cost Savings: Savings can be achieved by lighting some areas only when motion is detected.

\section{Historical Perspective}

Home Automation frequently relates to lighting control and specifically, the ability to dim lights. The dimming of light can be dated back to 1638 when Nicolla Sabbattini discovered that lowering cylindrical metal hoods around candles caused them to dim. Later, in 1814, the use of gas lighting with control valves provided a slightly more controlled dimming technique.

Saltwater became useful for dimming purposes in 1900. A tank was filled with saltwater and an electrode was submerged in one end of the tank. A second electrode was manually submerged and the depth of the submergence would determine how bright the lamp would be. Theatres would frequently have many of these tanks and the heat generated by the boiling saltwater would help heat the backstage areas. ${ }^{1}$ Resistance dimming with a rheostat was finally available in 1910. These dimmers were large, expensive, inefficient and generated a lot of heat. ${ }^{1}$

Silicon Controlled Rectifiers (SCRs) are the dimming method of choice in modern day dimming applications. Developed in 1958 by General Electric, SCRs are also called thyristors and operate by applying an external gate signal at timed intervals. Utilizing the SCR technique, home automation began to take shape in 1975 when the engineers at Pico Electronics began working on the X10 project (the project was their tenth project). ${ }^{2}$ Using Power Line Carrier Technology, or PLC, this technique is easy to implement for the installer, but the reliability of the system is often much less than desired. In recent years wired and wireless devices have been developed to compete with the X10 lineup, many with little success.

\section{Reinventing Home Automation}

The proposed home automation system is comprised of modules: a Master Control Module (MCM), one or more General Purpose Input Modules (GPIMs), one or more User Input Module (UIMs), and one or more Lighting Control Modules (LCMs). A programming module is also available which allows for plug-in firmware upgrades from a PC. An example of all modules interconnected is shown in figure 1.

The Master Control Module is responsible for coordinating communication between all of the other modules and the host PC (via RS-232). The MCM connects to the GPIMs and UIMs through a multi-node RS-485 network coined the "automation network." The LCMs connect via parallel ribbon cables.

The General Purpose Input Module is designed such that up to 16 devices can interface with the system. Engineered to allow modularity, each GPIM should be placed at a strategic location 


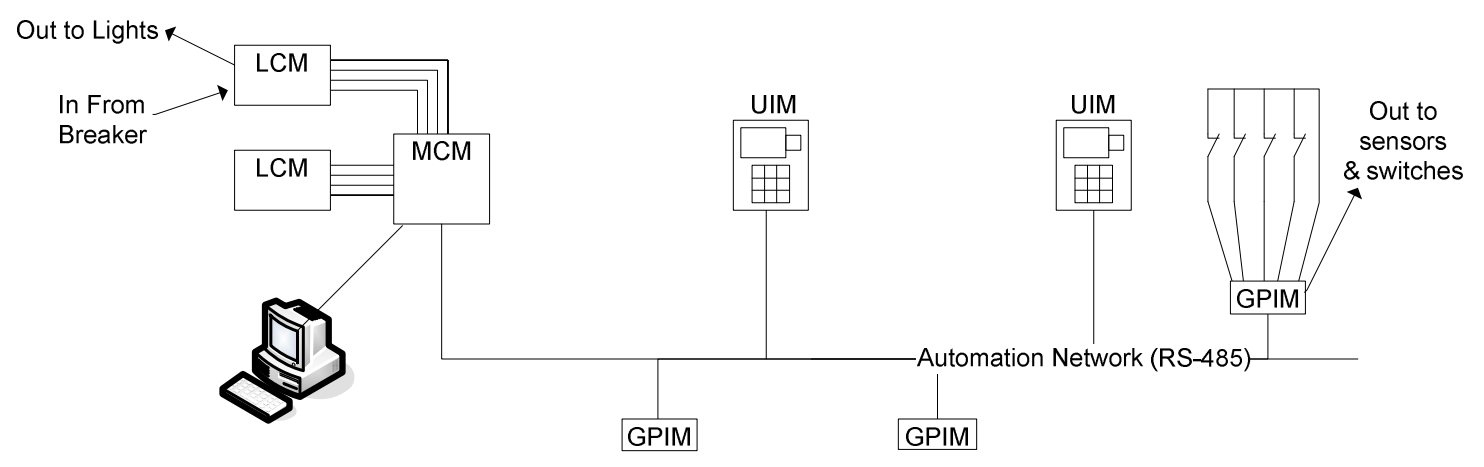

Figure 1: Full Home Automation System

within the home to minimize the wire required for implementation. The GPIM is bus-powered and requires an inexpensive, single 4-conductor twisted pair cable for operation. All inputs to the GPIM are standard dry-contact screw-terminal connections.

User Input Modules are available to allow users to interact with the system. These modules include a keypad for input and an LCD and relay for auxiliary output. The UIM may be used for arming and disarming the burglar alarm and for complete control over all lighting circuits.

The Lighting Control Module is responsible for the actual light dimming process. This is accomplished by sine-wave manipulation, a technique utilizing triacs triggering the source AC waveform at precisely the right time to create a new waveform. A triac is simply two SCRs connected back-to-back in a single package. This allows the triac to switch the waveform during both the positive and negative half-cycle. The LCM can dim 16 channels of lights in response to commands issued by the MCM (and ultimately from the host PC). This process is started by synchronizing with the incoming $60 \mathrm{~Hz}$ sine wave. Synchronization is achieved by detecting the zero-crossing point and triggering and external interrupt on the microcontroller. After successful synchronization, triacs connected to each channel are "fired" by a timer interrupt depending on the channel's intensity data. The MCM can control up to 4 LCMs (for a total of 64 lighting circuits).

The firmware for all modules of the automation system are written in ANSI C and compiled and linked with the Keil Cx51 compiler. To allow for fast application development, all host PC software is written in Visual Basic.NET. However, host PC software may be developed in any language that supports standard serial protocols.

\section{Master Control Module}

The MCM is responsible for facilitating communication between all devices within the system as well as with the controlling PC. As with all modules in this project, the MCM utilizes the Dallas DS89C450 microcontroller due to the inclusion of two onboard UARTs, 64kB of flash ROM, 8052 compatibility and built-in supervisory features. ${ }^{3}$ Providing +5 volts and +12 volts DC, the prototype uses a standard computer power supply to power the MCM and the devices on the automation network. 
By using both UARTs onboard the microcontroller, a connection to the automation network (via the RS-485 transceiver) and the host PC is possible. A connection to the host PC can be made directly or through the use of the programming interface (if firmware upgrades are necessary). The parallel interface of the MCM is designed to allow up to four LCMs to be connected.

The routines implemented in the firmware of the MCM represent a request-and-respond technique (the MCM requests data from each device on the automation network and waits for a response, or times out). This eliminates conflicts (collisions) on the network at the expense of network overhead.

\section{General Purpose Input Module}

The GPIM is connected at any point on the automation network and monitors up to 16 inputs. Up to 26 GPIMs can be used, allowing for up to 416 total inputs. Each input is a low-voltage, dry contact closure. The second UART on the DS89C450 is used simply for programming purposes (via the optional programming interface). The GPIM is powered by the automation network. At the MCM, the RS-485 bus is supplied with a 12-volt source. This higher voltage is necessary to allow for devices that need more than 5 volts to operate (such as passive infrared detectors, etc.). This will also compensate for any voltage drop that is observed on the RS-485 bus for a typical mid-sized home. The GPIM features a voltage regulator and filter circuit to reduce the source voltage down to 5 volts. Two LEDs are present; one for power and the other indicating network activity. The firmware of the GPIM is relatively simple as it only captures data on the ports when requested and sends the data back to the MCM.

\section{User Interface Module}

The UIM allows for users to interact with the system with a keypad and LCD display. The backlit LCD is a standard Hitachi HD44780 compatible model allowing for multiple sizes and configuration options. A firmware keypad decoding scheme is used for up to four rows and four columns. An auxiliary relay output is available that may be controlled locally or by the host PC.

The UIM firmware is the most complex of the project due to the task of allocating resources to the LCD, keypad and communication routines effectively. A user can arm and disarm the burglar alarm system as well as control all channels of lighting.

\section{Lighting Control Module}

The lighting control module (LCM) is responsible for dimming each lighting circuit in response to commands provided by the MCM. Dimming lighting loads is achieved by manipulating characteristics of the lighting circuit power signal using SCR/triac dimming. SCR/triac dimming achieves its goal by manipulating a waveform with phase control, while a resistive dimmer (such as a rheostat or autotransformer) dims by reducing the amplitude of a signal (lowering the voltage). The basic principle of the two techniques is the same; the lower the voltage, the dimmer the light. The difference comes in the way that the techniques achieve the lower effective voltage. Resistive dimming is the easier of the two. On the other hand, SCR/triac dimmers do not have the ability to directly reduce the amplitude of a signal. they can only turn 
on or off. Since traics are used more widely in dimming circuits than SCRs, the rest of the paper will mention triacs only. Triacs do not have the ability to be between the on and off state (allowing only "some" of the voltage to pass through). Ttraic dimming technique gets around this limitation by harnessing the shape of the source sine wave and using it as an advantage. With precise timing and synchronization, a lower average output voltage can be obtained by turning on the triac only during certain times in a single sine wave cycle. Figure 2 illustrates a simple modified sine wave (the shaded areas indicate an area of the sine wave that is switched on while the remainder is switched off).

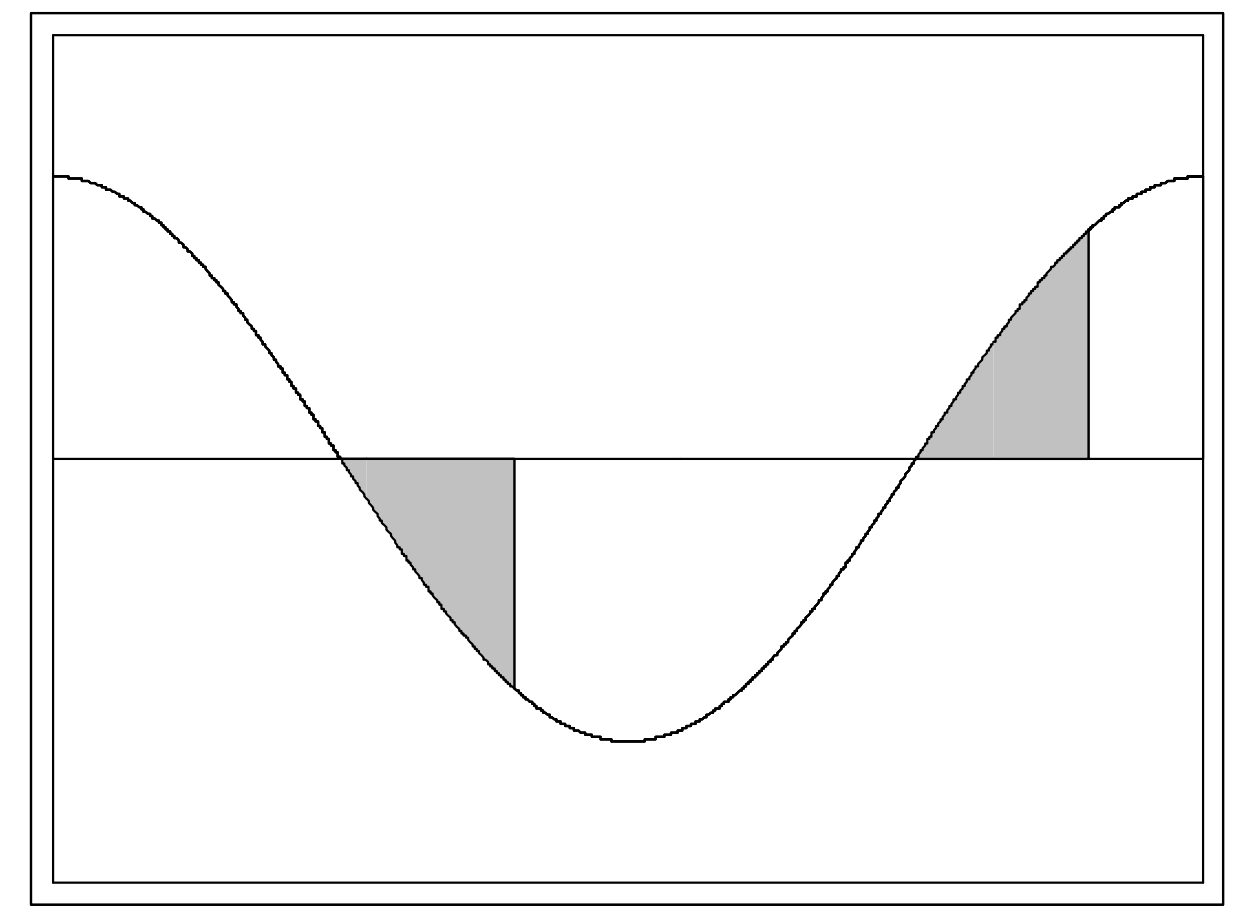

Figure 2: Modified Sine Wave

In order to prevent lamp flickering and to maintain a constant intensity it is important to synchronize the timing circuits responsible for firing the triacs with the incoming AC sine wave. This synchronization must be an ongoing procedure as any slight synchronization error will become visually apparent in the form of flickering or intensity "sway."

The LCM uses triacs triggered by the microcontroller. The microcontroller is synchronized by a zero-crossing detection circuit which simply trips an external interrupt at approximately the time the sine wave crosses the zero mark (twice a cycle).

The zero-crossing detector is responsible for sampling the incoming sine wave signal and producing a pulse when the signal crosses the zero reference point. This voltage is then fed into an external interrupt of the microcontroller. As seen in figure 3, the incoming sine wave (top waveform) has a corresponding trigger pulse (lower waveform) for each zero crossing point. 


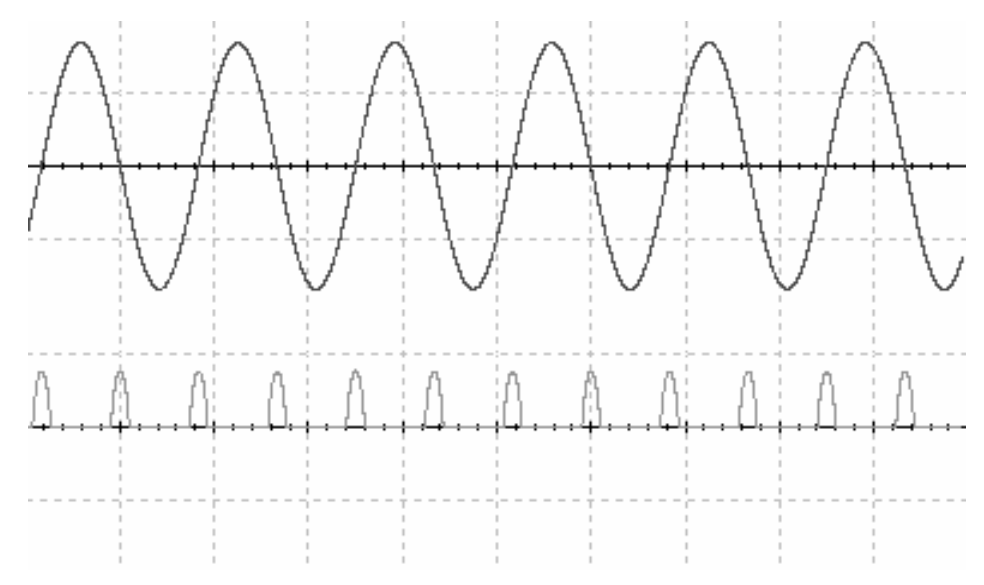

Figure 3: Zero Crossing Detection

The triac circuitry provides the high-speed switching capability necessary for lamp control and dimming. In its simplest form, a triac can be connected inline similar to a light switch and triggered with any low voltage source. ${ }^{3}$ The same technique holds true here, with the exception that the triac will be fired at high speed and with precision, all with the assistance of a microcontroller.

Communication is accomplished across a 1-byte data bus (P0), a 4-bit address bus (P3.4 - P3.7), an enable pin and a reset pin. While parallel bus technology is archaic with respect to modern serial interfaces, it is also easier to develop and much less demanding on the processor.

The firmware of the LCM includes the accurate timing necessary to achieve flicker-free dimmable lighting on 16 channels. All functions are called inside of interrupt service routines to allow for prioritization of the timing of the triac firing over communications.

Overload protection is mandatory. Each channel must be fused at no higher than 4A (fast blow) with the source circuits protected with a 15A (fast blow) fuse. Another safety consideration when working with a dimming system is the realization that not all lighting products are designed to be dimmed. Lighting channels one through four feature "Dim-Lock" jumpers that force the channel to be in only an on or off state (not dimmable).

The LCM requires two dedicated 15A source circuits, providing a total of 30A for each LCM in the system. Both source circuits must also be on the same phase to ensure accurate zero-crossing detection

\section{Programming Interface}

This interface allows a PC to communicate directly with modules and update the firmware as needed. The LCM does not feature a header connector and is not in-field firmware upgradeable. 


\section{Host PC Software}

The host PC, ultimately responsible for coordinating all of the events of the automation system, is running an application written in Visual Basic.NET. The prototyped application includes a manual control interface as shown in figure 4 . This allows control of all modules on the automation network as well as control of the first eight channels of light on the first LCM (only eight lamps are included on the demonstration board, making 16 dimmer controls unnecessary in this demonstration application).

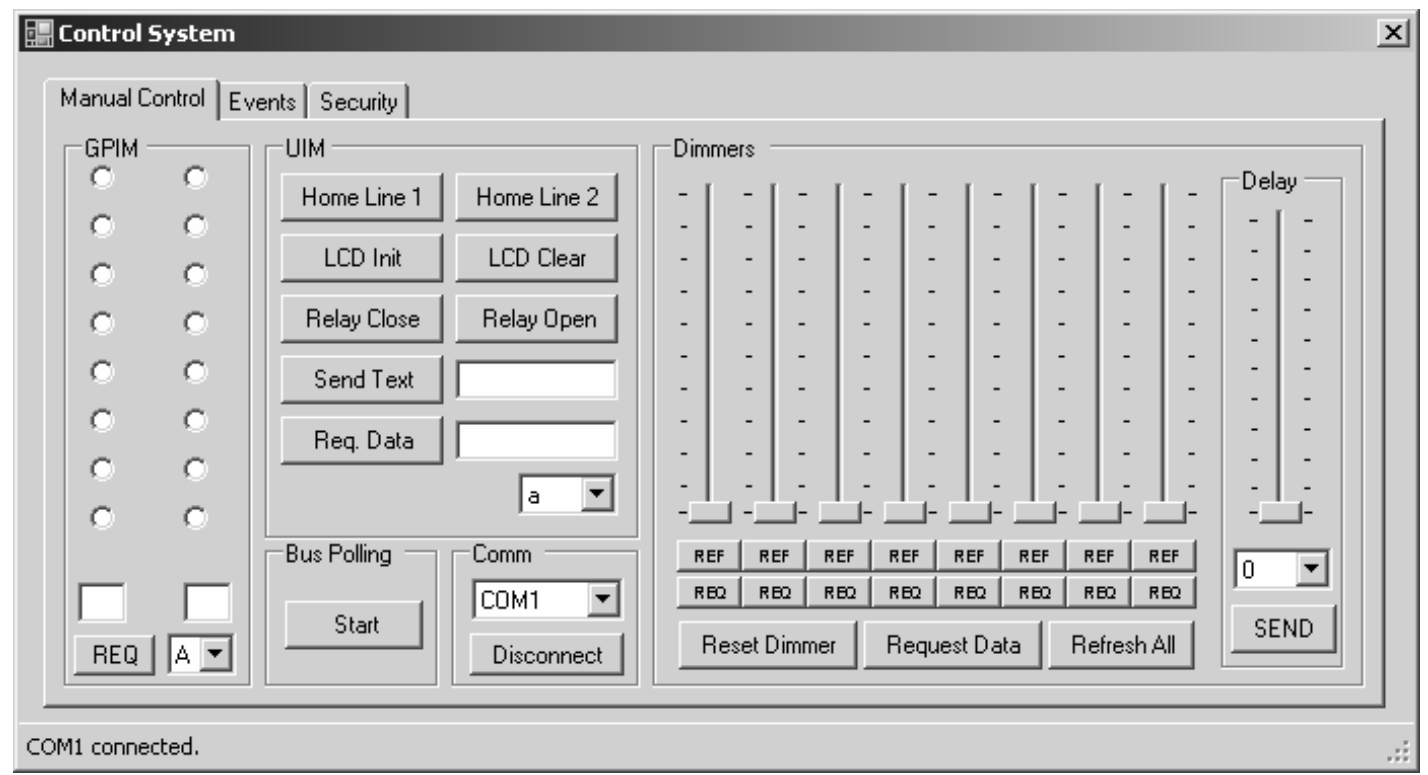

Figure 4: Manual Control Screen

The manual control system is mainly designed for debugging purposes. The benefit of this system comes with event processing. In figure 5, the event list is shown. This allows the system to respond to events triggered by inputs connected to the GPIM(s).

The list contains a name and description of each event to aid the user as the event list will probably have many events for an average size home. Adding and editing an event is very simple using the built-in event editor. The system also has safeguards to prevent edit entry mistakes.

The security tab, shown in figure 6, displays configuration parameters for the security system. Modeled after standard security systems, the security interface features multiple user codes with logging capabilities, delayed entry and exit zones with courtesy light controls and follower zones with automatic occupant detection.

The Visual Basic implementation of the software includes a custom communications module designed specifically to interface with the MCM. This module can be imported into any .NET project (including other languages supported by the .NET framework) allowing for easy customization for any application. 


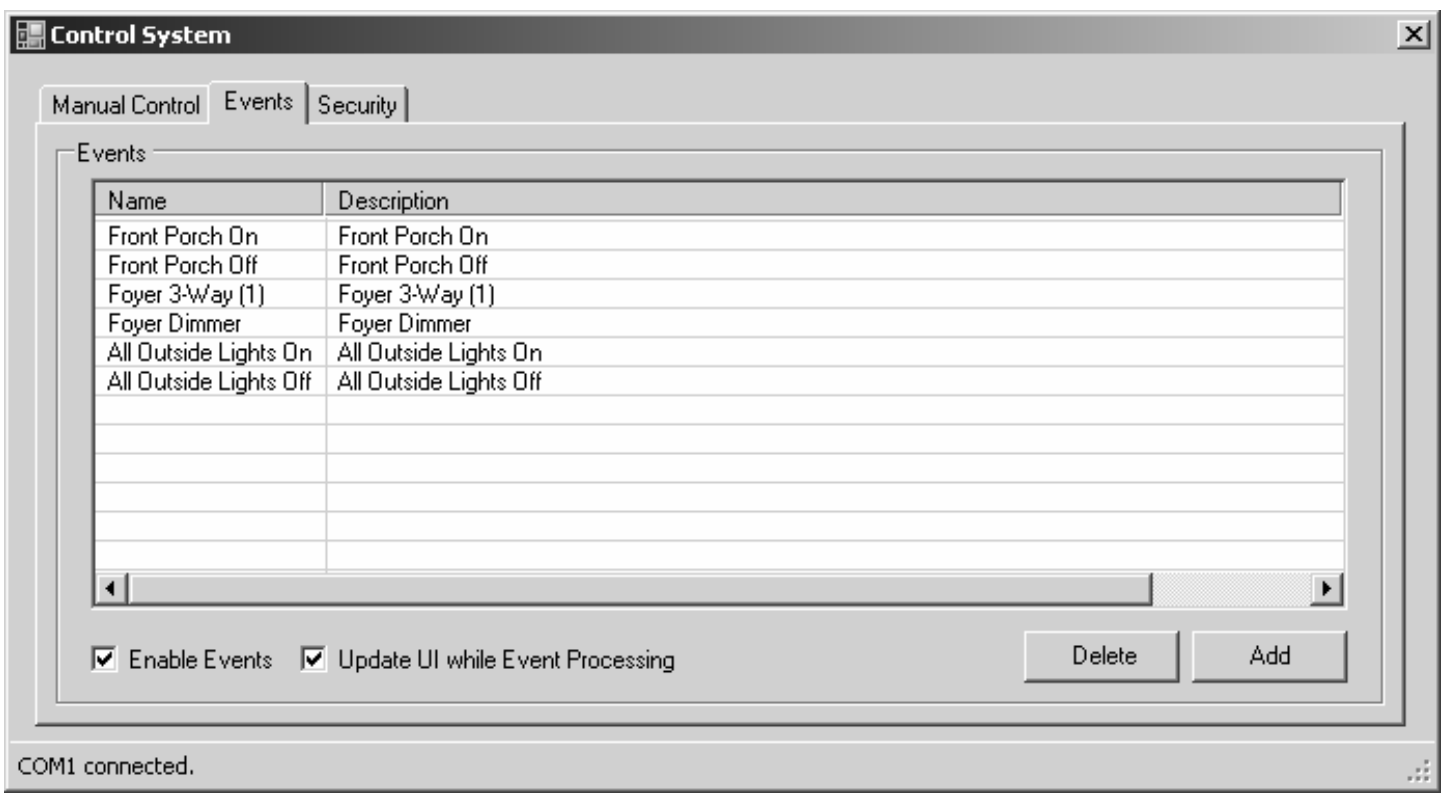

Figure 5: Events List Screen

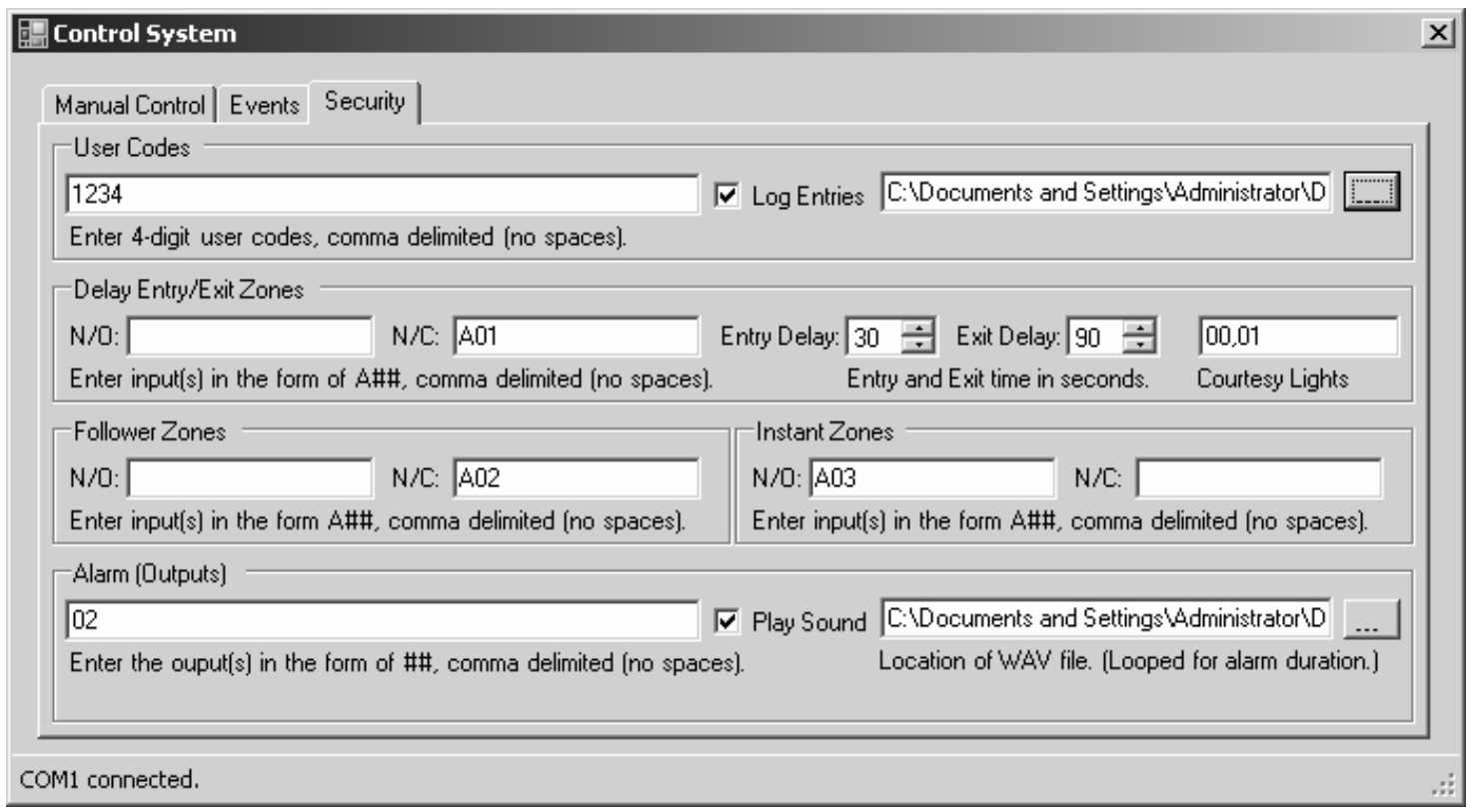

Figure 6: Security Screen

\section{Future Considerations}

The host PC software must be modified to include an event-driven technique to acquire data from the GPIM and UIM devices. This may lower the resource demands of the application as the polling technique requires a large amount of resources from the host PC. The manual control 
system should be upgraded to allow full control of multiple LCMs. Scheduled triggering of events would allow for vacation timing and other useful timed events to occur without the need for manual triggers. The security system should include the ability to drive another form of output in the event of an alarm (siren, etc.). The system has multiple input interfaces but is limited to lighting for output. Further testing should be done to prevent interference on the automation network and the connected modules.

The host PC software is the logical environment to include DTMF signaling and Internet connectivity as well as other communication techniques. Configuration and add-on opportunities are endless.

\section{Conclusions}

The proposed home automation system presented in this paper was designed, built, and successfully tested. As a result, home automation devices can be designed in a modular fashion so that reliable, cost effective and upgradeable systems can be made available to the masses. This can be achieved by reinventing the residential dimmer to support only the amount of wattage that is needed and hardwire the remaining portions of the system with inexpensive lowvoltage cable and devices. This also allows for easy configuration changes that the home occupant can accomplish without professional assistance, allow for built-in security features, and potential cost savings.

The above paper is the result of a research thesis presented to the graduate faculty of Middle Tennessee State University in partial fulfillment of the Master of Science degree in Engineering Technology. The research has enhanced the real-world applications and hands-on experiences of the author.

\section{Bibliography}

1. Williams, B. (2005). A History of Light and Lighting. Retrieved September 15, 2006 from http://www.mts.net/ william5/history/hol.htm

2. Driscoll, E. B. (2002). A Timeline for Home Automation. Retrieved September 20, 2006 from http://www.eddriscoll.com/timeline.html.

3. Maxim Integrated Products, Inc., A Dallas Semiconductor Corporation. (2005). DS89C430/DS89C440/89C450 Ultra-High-Speed Flash Microcontrollers. Sunnyvale, CA.

4. Goldwasser, S. M. (2006). Sam's Schematic Collection: Simplest Dimmer Schematic. Retrieved September 15, 2006 from http://www.repairfaq.org/sam/samschem.htm\#schsld 